\title{
The role of nucleus accumbens shell in learning about neutral versus excitatory stimuli during Pavlovian fear conditioning
}

\author{
Laura A. Bradfield and Gavan P. McNally ${ }^{1}$ \\ School of Psychology, The University of New South Wales, Sydney 2052, Australia
}

\begin{abstract}
We studied the role of nucleus accumbens shell (AcbSh) in Pavlovian fear conditioning. Rats were trained to fear conditioned stimulus A (CSA) in Stage I, which was then presented in compound with a neutral stimulus and paired with shock in Stage II. AcbSh lesions had no effect on fear-learning to CSA in Stage I, but selectively prevented learning about the neutral conditioned stimulus (CS) in Stage II. These results add to a growing body of evidence indicating an important role for the ventral striatum in fear-learning. They suggest that the ventral striatum and AcbSh, in particular, directs learning toward or away from a CS as a consequence of how well that CS predicts the shock unconditioned stimulus (US). AcbSh is required to reduce the processing of established predictors, thereby permitting neutral or less predictive stimuli to be learned about.
\end{abstract}

The nucleus accumbens (Acb) is a complex and heterogeneous structure located in the ventral striatum. In the rat, Acb can be divided into at least two main compartments: a central core (AcbC) and a surrounding shell (AcbSh). AcbSh itself can be subdivided into dorsomedial, ventral, and lateral regions. Acb is located centrally within the mesolimbic pathway and is thought to be the motor-limbic interface (Mogenson 1987). Although typically associated with reward learning and appetitive motivation (e.g., Knuston and Cooper 2005; Wise 2006; Day and Carelli 2007), there is evidence that Acb is important for Pavlovian fear conditioning. This includes demonstrations that extracellular dopamine in the AcbSh, but not AcbC, increased in response to aversive events such as restraint (Deutch and Cameron 1992) and footshock (Kalivas and Duffy 1995), as well as to an auditory conditioned stimulus (CS) signaling footshock. Importantly, this role for Acb in fear-learning is independent of appetitive motivation (Josselyn et al. 2005).

Lesion and microinjection approaches have been used to study the role of Acb in Pavlovian fear conditioning. The results of these studies have typically been interpreted to mean that AcbSh contributions depend on the modality of the CS. For example, Riedel et al. (1997) reported that AcbSh lesions impaired context fear-learning but spared fear-learning to a discrete CS. Likewise, Jongen-Relo et al. (2003) reported that AcbSh lesions impaired context but not CS fear-learning, and further found that AcbC lesions had no significant effect on either type of learning. Finally, Westbrook et al. (1997) reported that a microinjection of morphine into Acb impaired fear-learning to contextual but not discrete CSs. These findings have been taken as evidence for a role of AcbSh in contextual fear-learning. However, these dissociations between contextual and discrete fear-learning have not always been replicated. For example, Parkinson et al. (1999) found that AcbC lesions affected both context and discrete CS fear, whereas AcbSh lesions affected neither.

It has been suggested that procedural differences, such as lesion size and type, may contribute to these inconsistent effects

'Corresponding author.

E-mail g.mcnally@unsw.edu.au; fax 61-2-93853641.

Article is online at http://www.learnmem.org/cgi/doi/10.1101//m.1798810. of AcbSh manipulations on context versus discrete fear-learning. However, there is a theoretically more interesting and significant possibility. In many of these studies, the manipulations (lesion, microinjection) did not affect fear-learning to a discrete CS. In addition, deficits in contextual fear-learning were only observed under conditions where both the context and discrete CS were co-present at reinforcement (i.e., delay conditioning to a discrete CS) (e.g., Riedel et al. 1997; Jongen-Relo et al. 2003).Thus, rather than being important for learning about CSs of different modalities (contextual versus discrete), AcbSh may be important for learning about CSs based on their status as reliable predictors of the US. In standard delay conditioning, the context is a poorer predictor of the US than the discrete CS and AcbSh lesions typically impair contextual fear-learning under these conditions. When the context is arranged to be a better predictor of the US than a discrete CS (e.g., trace fear conditioning), AcbSh lesions do not affect learning (Parkinson et al. 1999). Further, AcbSh lesions impair fear-learning to a discrete auditory CS when it is a poor predictor (such as in trace conditioning), but not when that CS is a good predictor (such as in delay conditioning) of the US (Cassaday et al. 2005).

The experiments reported here were designed to test the possibility that AcbSh is important for fear-learning not because it contributes to learning about CSs on the basis of their modality (contextual versus discrete), but instead because it selects individual CSs for fear association on the basis of their status as reliable predictors of an aversive US. In these experiments, rats received excitotoxic or sham lesions of AcbSh prior to fear conditioning. In Stage I, rats received fear conditioning of CSA to establish it as a reliable predictor of the aversive US. In Stage II, rats received compound fear conditioning of the predictive (excitatory) CSA and a nonpredictive (neutral) CSB. Across the experiments we varied the nature of Stage I and Stage II training to study the role of AcbSh in learning about the predictive and nonpredictive stimuli during Stage II. However, a fundamental difficulty with this approach, and any approach that studies learning about co-presented stimuli differing in their training history (e.g., contextual versus discrete fear conditioning), is that a predictive CS will necessarily evoke more responding than a less predictive CS. This renders inferences about how any manipulation affects 
learning about these CSs difficult. These experiments therefore used a testing procedure that allowed us to determine how much was learned about CSA and CSB, relative to each other, during Stage II.

\section{Experiment 1}

Experiment 1 comprised a discrimination task that initially established a discrete CS as a reliable predictor of danger in Stage I, but then required rats to ignore the predictive CS in Stage II and instead learn about initially neutral (i.e., initially nonpredictive) CSs. The design is shown in Table 1 . Rats were trained to fear CSA in Stage I. This training was intended to establish CSA as predictive of shock. In Stage II, rats received $\mathrm{AB}+/ \mathrm{AC}-$ discrimination training. To learn this discrimination rats are required to shift attention away from the previously predictive CSA toward CSB and CSC. It was expected that sham animals would solve this discrimination and show more fear of $\mathrm{AB}$ than $\mathrm{AC}$ on test. The question was how AcbSh lesions would affect this discrimination learning.

\section{Histology}

Histological analysis determined the size and placement of lesions. Figure 1A shows a representation of the minimal (black) and the maximal (gray) extent of AcbSh lesions. The lesions were uniform in that they targeted medial sections of the AcbSh, and never the lateral section. In addition, lesions tended to target more caudal regions of the AcbSh with the rostral pole almost never affected. Occasionally a small section of the AcbC was affected. A total of five animals were excluded from the experiment because of incorrect lesion placement or size. Thus, 17 animals were included in the analysis (Sham, $n=9$, Lesion, $n=8$ ).

\section{Behavior}

The mean and SEM levels of freezing to CSs across the course of the experiment are shown in Figure 1B. Mean levels of freezing during the first $3 \mathrm{~min}$ of context alone were as follows: Sham group: Stage $\mathrm{I}=13 \%(\mathrm{SEM}=6)$, Stage $\mathrm{II}=7 \%(\mathrm{SEM}=4)$, Test $=$ $16 \%(\mathrm{SEM}=5)$; Lesion group: Stage I $=10 \%(\mathrm{SEM}=5)$, Stage $\mathrm{II}=11 \%(\mathrm{SEM}=5)$, Test $=5 \%(\mathrm{SEM}=4)$. Inspection of the figure suggests that there were no differences in freezing between the groups during Stage I. The figure also suggests that there were no differences in freezing to $\mathrm{AB}$ and $\mathrm{AC}$ during Stage II, either within or between groups. Finally, it is clear from the figure that the sham group acquired the discrimination and were more fearful of $\mathrm{AB}$ than $\mathrm{AC}$ on test, whereas the lesioned group did not solve this discrimination and were equally afraid of $\mathrm{AB}$ and $\mathrm{AC}$. This failure of discrimination learning was due to the lesioned group being less afraid than shams of $\mathrm{AB}$. The groups did not differ in fear of AC.

During each stage of the experiment, levels of freezing to the CS were significantly greater than during the first 3 min of context alone, $F \mathrm{~s}_{(1,15)}=32.1,77.6$, and $97.5, P<0.05$ for Stage I, Stage II, and Test, respectively. There were no preCS $\times$ Group interactions

Table 1. Summary of experimental designs

\begin{tabular}{lclc}
\hline Experiment & Stage I & Stage II & \multicolumn{1}{c}{ Test } \\
\hline 1 & $A+$ & $A B+/ A C-$ & $A B$ versus $A C$ \\
2 & $A+$ & $A B+$ & $A D$ versus $B C$ \\
& $C+$ & & \\
\hline
\end{tabular}

In each experiment there were AcbSh sham and AcbSh lesion groups. A, B, $C$, and D were four conditioned stimuli, a clicker, tone, light, and flashing light (counterbalanced); + denotes a $0.4 \mathrm{~mA}$ shock US.
A
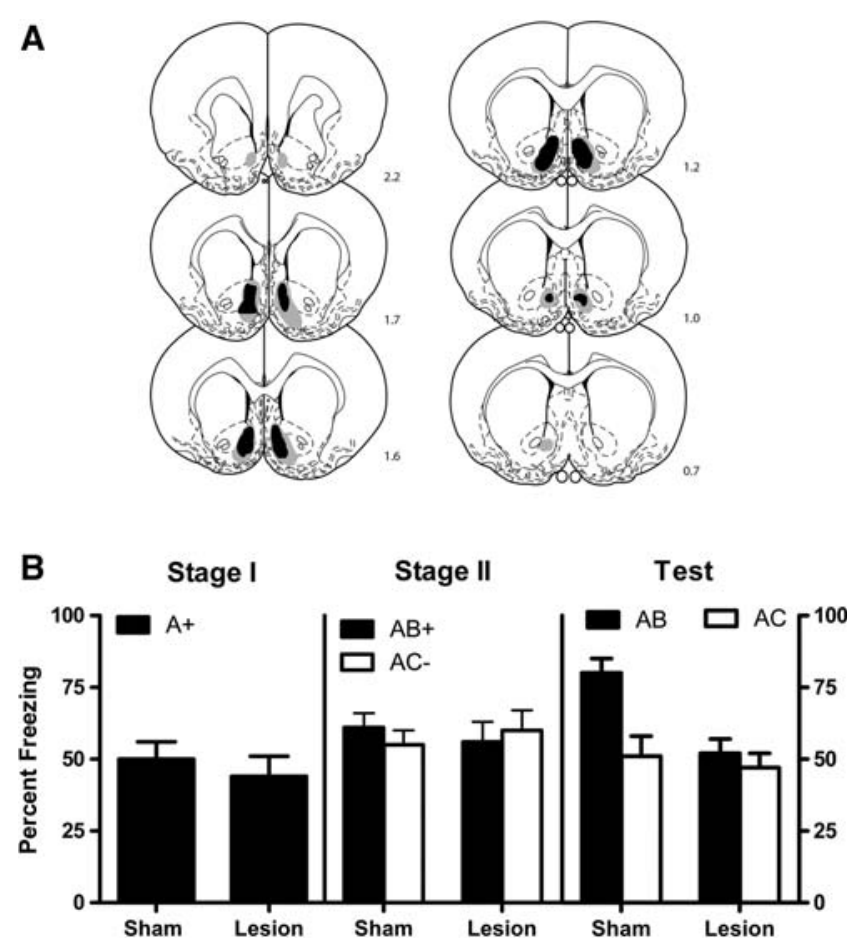

Figure 1. (A) Minimal (black) and maximal (gray) extent of NMDA-induced excitotoxic AcbSh lesions in Experiment 1. (B) Freezing during Stages I and II and Test for Experiment 1. AcbSh lesions prevented learning about the added neutral $C S s$ and $C: A B=A C$ on test.

at any stage: $F \mathrm{~s}_{(1,15)}<1, P>0.05$ for each stage. At the end of Stage I, there was no difference between sham and lesion groups in levels of freezing to A, $F_{(1,15)}<1, P>0.05$. At the end of Stage II there was no difference between sham and lesion groups in overall levels of freezing averaged across CS type, $F_{(1,15)}<1$, $P>0.05$, no difference in freezing to $\mathrm{AB}$ and $\mathrm{AC}$ averaged across group, $F_{(1,15)}<1, P>0.05$, and no Group $\times$ CS type interaction $F_{(1,15)}=2.4, P>0.05$, indicating that each group froze equally to each CS. The simple effects analysis revealed that on test, the sham group displayed greater freezing to $\mathrm{AB}$ than $\mathrm{AC}, F_{(1,15)}=$ 21.4, $P<0.05$, whereas the lesioned group did not, $F_{(1,15)}<1$, $P>0.05$. Further simple effects analysis revealed that the sham group froze more to $\mathrm{AB}$ than the lesioned group, $F_{(1,15)}=13.2$, $P<0.05$, but the groups did not differ in levels of freezing to AC, $F_{(1,15)}<1, P>0.05$.

These results show that after Stage I A+training, animals with sham lesions of AcbSh could learn the $\mathrm{AB}+/ \mathrm{AC}-$ discrimination in Stage II because they displayed less fear to $A C$ than $A B$ on test. This pattern of responding was not detected during Stage II, but it should be noted that shock was still present during Stage II while testing was conducted in extinction. This shows that sham lesioned animals could learn about the added neutral CSs during Stage II. In marked contrast, animals with AcbSh lesions did not learn this discrimination, instead displaying equivalent levels of fear to $\mathrm{AB}$ and $\mathrm{AC}$. Indeed, the performance on test of AcbSh lesioned animals was consistent with AcbSh lesioned animals learning exclusively about CSA to the detriment of CSs B and C. This would resemble partial reinforcement of CSA (i.e., A+ in Stage I then A+/A - in Stage II) causing reduced fear to CSA. Regardless, the failure of AcbSh lesioned rats to learn the $\mathrm{AB}+/ \mathrm{AC}-$ discrimination suggests that the AcbSh lesions impaired fear-learning about the initially neutral CSs, B and $\mathrm{C}$. 


\section{Experiment 2}

The results of Experiment 1 suggest that AcbSh lesions impair fearlearning to neutral CSs when conditioned in compound with an excitatory or already predictive CS. However, other interpretations are possible. For example, AcbSh lesioned rats may not have been able to discriminate between $\mathrm{AB}$ and $\mathrm{AC}$ causing them to freeze equally to each compound CS on test. Further, Experiment 1 did not directly compare how much was learned about the predictive CSA relative to the neutral CSs, B and C, during compound conditioning in Stage II. Experiment 2 addressed these issues.

The difficulty in directly assessing how much is learned about a predictive CS relative to a neutral CS when they are conditioned together is that the predictive CS will necessarily evoke more conditioned responding on test due to its prior training. This differential location of the two CSs on the performance scale means that simply comparing levels of freezing to them on test is uninformative regarding how much was actually learned about them during their compound conditioning. We employed a compound test procedure created by Rescorla (2001) to specifically address this problem. The design is shown in Table 1. CSA and CSC were separately paired with shock during Stage I. In Stage $\mathrm{II}$, rats received presentations of the compound $\mathrm{AB}$, also paired with shock. The question of interest was whether sham rats would learn equally or unequally about the predictive CSA and the nonpredictive CSB during Stage II, and how AcbSh lesions would affect this learning. Performance could not be tested to CSA and CSB alone because the excitatory CSA would necessarily evoke more freezing on test than CSB, regardless of how much was learned about each during Stage II. Instead, rats were tested for fear responses to a compound of CSA with the untrained CSD (AD) and a compound of CSB with CSC (BC). The logic of this procedure is this: If these compounds were tested at the end of Stage I, responding to $\mathrm{AD}$ and $\mathrm{BC}$ would be equivalent because each was composed of a CS that had been reinforced in Stage I (A and C) and a CS that had not (B and D). As such, these compounds equate Stage I training so that any performance differences to $\mathrm{AD}$ and $\mathrm{BC}$ on test can only be a result of different amounts being learned about CSA and CSB during Stage II. Under such conditions, more is learned about the nonpredictive CSB than the predictive CSA during Stage II and thus responding to BC is greater than AD on test (Rescorla 2001; Bradfield and McNally 2008). The question was how AcbSh lesions would affect this learning.

\section{Histology}

Figure 2A shows a representation of the minimal (black) and the maximal (gray) extents of AcbSh lesions. A total of four animals were excluded from the experiment because of incorrect lesion placement or size. Thus, 20 animals were included in the analysis (Sham, $n=12$, Lesion, $n=8$ ).

\section{Behavior}

The mean and SEM levels of freezing to CSs across the course of the experiment are shown in Figure 2B. Mean levels of freezing during the first $3 \mathrm{~min}$ of context alone were as follows: Sham group: Stage $\mathrm{I}=1 \% \quad(\mathrm{SEM}=1)$, Stage $\mathrm{II}=12.5 \% \quad(\mathrm{SEM}=5)$, Test $=6 \%(\mathrm{SEM}=3)$, Lesion group: Stage $\mathrm{I}=1 \%(\mathrm{SEM}=1)$, Stage $\mathrm{II}=12 \%(\mathrm{SEM}=3.5)$, Test $=9 \%(\mathrm{SEM}=4)$. Inspection of the figure suggests that there was no difference in freezing between groups or CS type (A versus C) during Stage I. The figure also suggests that there were no differences in freezing between groups in Stage II to AB. Finally, it is clear from the figure that sham rats froze more to $\mathrm{BC}$ than $\mathrm{AD}$, but lesioned rats froze more to $\mathrm{AD}$ than $\mathrm{BC}$ on test.
A
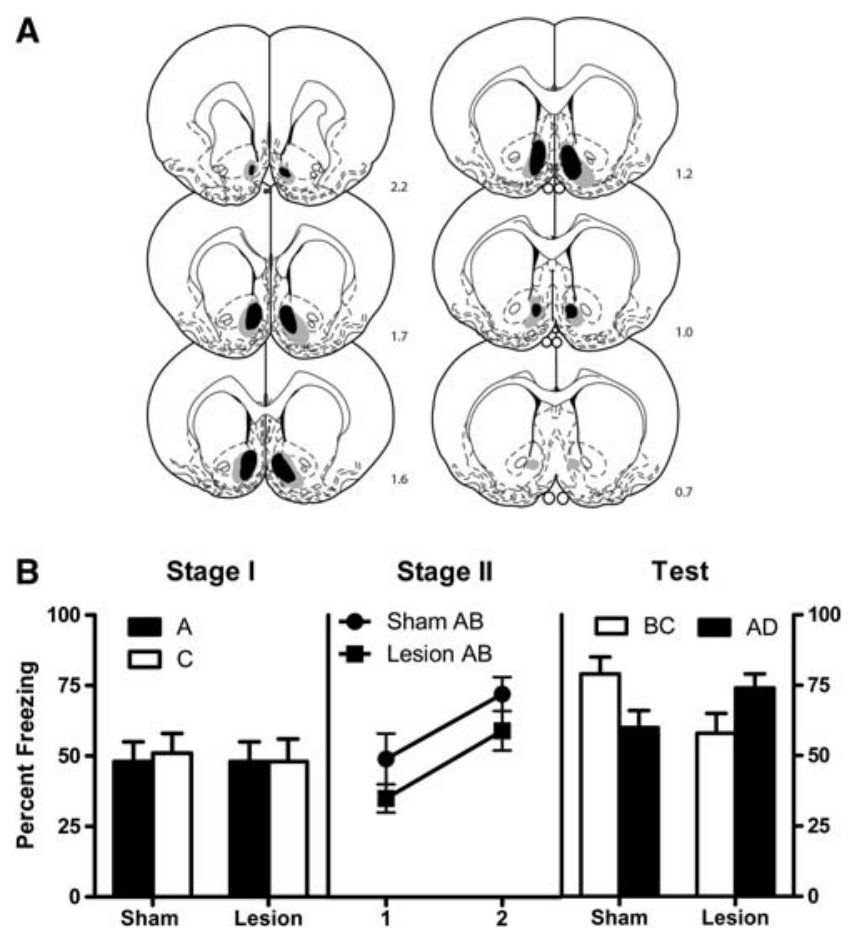

Figure 2. (A) Minimal (black) and maximal (gray) extents of NMDA-induced excitotoxic AcbSh lesions in Experiment 2. (B) Freezing during Stages I and II and Test for Experiment 2. The AcbSh lesions augmented learning about the pretrained fear CS (A) relative to learning about the added neutral $C S(B): A D>B C$ on test.

During each stage of the experiment levels of freezing to the CS were significantly greater than during the first 3 min of context alone, $F \mathrm{~s}_{(1,18)}=131.5,84.5$, and 201, $P<0.05$ for Stages I and II and Test, respectively. There were no preCS $\times$ group interactions at any Stage: $F \mathrm{~s}_{(1,18)}<2, P>0.05$ for each stage. At the end of Stage I, there was no difference between sham and lesioned rats in overall levels of freezing averaged over CS type, $F_{(1,18)}<1$, $P>0.05$, no difference in overall levels of freezing to CSA and CSC averaged over groups, $F_{(1,18)}<1, P>0.05$, and no interaction between CS type and group, $F_{(1,18)}<1, P>0.05$, indicating that each group froze equally to each CS. During Stage II there was no difference between sham and lesioned rats in levels of freezing to $\mathrm{AB}, F_{(1,18)}=1.5, P>0.05$. Simple effects analysis revealed that on test, sham rats froze more to $\mathrm{BC}$ than $\mathrm{AD}$, $F_{(1,18)}=11.2, P<0.05$, whereas lesioned rats froze more to AD than BC, $F_{(1,18)}=5.5, P<0.05$. Further simple effects analysis revealed that sham rats froze more to $\mathrm{BC}$ than lesioned rats, $F_{(1,18)}=4.6, P<0.05$, but sham and lesioned rats froze equally to $\mathrm{AD}, F_{(1,18)}=2.6, P>0.05$. These simple effects comprise a significant group $\times$ CS type interaction, $F_{(1,18)}=15.39, P<0.05$.

These results confirm previous findings (Rescorla 2001; Bradfield and McNally 2008) that more is learned about the less predictive $\mathrm{CSB}$ than the predictive $\mathrm{CSA}(\mathrm{BC}>\mathrm{AD})$ when the two CSs are reinforced in compound. AcbSh lesions reversed this normal profile of learning so that $\mathrm{AD}>\mathrm{BC}$ on test for lesioned animals. This shows directly that AcbSh lesions cause animals to learn more about a predictive CSA at the expense of a less predictive CSB.

\section{Discussion}

These experiments studied the role of AcbSh in Pavlovian fear conditioning using compound conditioning designs. The aim 
was to test the possibility that AcbSh may be important for fearlearning based on the predictive status of a CS. Experiment 1 asked whether rats with AcbSh lesions could solve a discrimination that explicitly required learning about added, neutral CSs at the expense of a pretrained predictive CS. They could not. Thus, whereas sham lesioned animals could use the added neutral stimuli (B and $\mathrm{C}$ ) to acquire the $\mathrm{AB}+/ \mathrm{AC}-$ discrimination, AcbSh lesioned rats showed a pattern of responding that was consistent with the exclusive learning about CSA. Experiment 2 used a compound test procedure to directly assess how much was learned about a predictive CS and a neutral CS during compound conditioning. The results showed that more was learned about the predictive CSA than the neutral CSB and this profile of learning was opposite to that observed in sham animals. Taken together, these experiments identify an important and novel role for AcbSh in Pavlovian fear conditioning, specifically in the distribution of learning among competing predictors of danger.

In the experiments reported here AcbSh lesions had no effect on fear-learning to a discrete CS during Stage I training when that CS was conditioned in isolation. This is consistent with past research that has likewise failed to detect an effect of AcbSh lesions on conditioning to single, discrete CSs. This is important because it shows that fear-learning and memory consolidation to a single CS can occur independently of the AcbSh. Rather, AcbSh lesions acted selectively to affect fear-learning during compound conditioning of CSs that differed in their predictive status. Specifically, during compound conditioning of a neutral and predictive CS, AcbSh lesions reduced or prevented learning about the neutral CS relative to the amount that was learned about the pretrained, predictive CS. This profile of learning was different to that observed in sham lesioned animals. Notably, sham lesioned rats learned more about the neutral CS than the pretrained CS. Expressed differently, rats with sham lesions learned more about the least predictive CS (the CS with the larger individual prediction error) whereas AcbSh lesioned rats learned more about the most predictive CS (the CS with the smaller individual prediction error). This suggests that AcbSh is normally required to reduce processing of established predictors, thereby permitting neutral or less predictive stimuli to be learned about. Lesions of AcbSh prevent this reduction in processing of established predictors and therefore promote more learning to them.

The neural circuitry enabling this role for AcbSh in reducing processing of established predictors is unclear. AcbSh receives extensive projections from medial prefrontal cortex, basolateral amygdala, hippocampus, and midline thalamus. The hippocampal input to AcbSh via subiculum (Groenewegen et al. 1987) is of potential importance. Hippocampal lesions, like AcbSh lesions, can prevent latent inhibition (Kaye and Pearce 1987; Han et al. 1995) and also prevent the reduced processing of established predictors (Han et al. 1995). Thus, a hippocampus to AcbSh pathway may be critical in regulating how much is learned about competing predictors of danger. This possibility warrants empirical attention as does whether this role for AcbSh is specific to fear conditioning.

Our results are consistent with the effects of AcbSh lesions on contextual versus discrete CS fear conditioning (i.e., Riedel et al. 1997; Parkinson et al. 1999; Jongen-Relo et al. 2003). As reviewed previously, it is commonly observed that AcbSh lesions impair fear-learning to contextual, but not discrete, CSs when those discrete CSs are subjected to delay fear conditioning. This has been interpreted to mean that AcbSh is especially important for contextual fear-learning. However, under such circumstances, the background contextual cues are also poorer predictors of the US than the discrete CS due to, in part, differences in salience but also differences in their temporal properties. For example, contextual cues have more opportunity for extinction in the intertrial interval. These differences in salience and associative strength are difficult to control for or make explicit. The experimental procedures used here made explicit these differences in associative strength by studying learning about CSs that were trained to different levels of associative strength prior to compound conditioning. We then employed test procedures designed to assess how much was learned about these CSs when they were conditioned in compound. Thus, the results of these experiments underscore the usefulness of the compound test procedure (Rescorla 2001) in studying the neural mechanisms of fear-learning.

It is worth comparing our findings with those reported in latent inhibition preparations. In a typical latent inhibition experiment, subjects receive nonreinforced exposures to the to-be-conditioned stimulus. This CS is then paired with shock. There is typically less responding to the CS on test among groups that received CS pre-exposure compared to groups that did not. AcbSh lesions consistently disrupt such latent inhibition. AcbSh lesions augment fear conditioning to the pre-exposed CS relative to sham lesions (Weiner et al. 1996, 1999; Jongen-Relo et al. 2003; Gal et al. 2005). Our analysis suggests a reinterpretation of this role for AcbSh in latent inhibition. During the typical latent inhibition experiment, there are at least two stimuli competing to enter into associations with the shock US: the target preexposed CS and the nontarget pre-exposed background contextual cues. The pre-exposed CS, when paired with the US, remains a better predictor of shock than the pre-exposed context. Thus, the disruption of latent inhibition by AcbSh lesions could be reinterpreted as indicating that AcbSh lesions augment learning about the best predictor of shock (the discrete CS) at the expense of the poorer predictor (the context).

It is also worth commenting on the potential effects of AcbSh lesions that might confound interpretation of these data. AcbSh lesions can increase locomotor activity (Weiner et al. 1996; Jongen-Relo et al. 2003). It is possible that increased locomotor activity could interfere with expression of defensive behaviors such as freezing. Although we did not assess locomotor activity in these experiments there are two reasons why potential changes in locomotor activity are unable to account for our results. First, the experiments reported here used a within-subject design to compare performances to the target CSs on test. Locomotor activity was therefore matched across stimuli. Second, each of the studies that have reported an effect of AcbSh lesions on locomotor activity measured this activity in an open field (Weiner et al. 1996; Jongen-Relo et al. 2003). This contrasts with the current study, which measured freezing in conditioning chambers that offered relatively restricted space. Maldonado-Irizarry and Kelley (1995) also measured locomotor activity in a confined activity cage and found no differences between AcbSh lesioned and sham rats.

These experiments add to a growing body of evidence (for review, see McNally and Westbrook 2006) suggesting extension to contemporary accounts of the neural mechanisms of fearlearning. The predominant model of the neural mechanisms of fear-learning emphasizes the role of amygdala in the formation, storage, and expression of fear associations (e.g., Pare et al. 2004; Fanselow and Poulos 2005; Maren 2005). There is a wealth of data in support of this model. Nonetheless, there is increasing evidence that fear-learning, in both rodents and humans, depends on structures other than amygdala. The present experiments identify a key role for AcbSh in fear conditioning. Human functional magnetic resonance imaging (fMRI) studies frequently detect significant blood-oxygen-level-dependent (BOLD) signals in ventral striatum correlated with aversive learning (e.g., Seymour et al. 2004; Delgado et al. 2008). Concerns whether rodent and human striatal regions are homologous render comparisons of experimental results between species difficult. 
Nonetheless, taken together, these findings are consistent with the possibility that ventral striatum is critically involved in the stimulus selection process that distributes learning between competing predictors of danger. The ventral striatum and AcbSh, in particular, directs learning toward or away from a CS as a consequence of how well that CS predicts the shock US. AcbSh is required to reduce processing of established predictors thereby permitting neutral or less predictive stimuli to be learned about.

\section{Materials and Methods}

\section{Subjects}

The subjects were experimentally naïve male Wistar rats weighing between 280 and $320 \mathrm{~g}$ at the start of the experiment. Prior to surgery there were 22 rats in Experiment 1 and 24 rats in Experiment 2. Rats were obtained from a commercial supplier (Monash Animal Services). They were housed in groups of eight in plastic boxes $(67 \mathrm{~cm}$ in length, $40 \mathrm{~cm}$ in width, and $22 \mathrm{~cm}$ in height) with food and water continuously available. The boxes were kept in an air-conditioned colony room maintained under natural lighting. Each rat was handled for $3 \mathrm{~d}$ prior to the start of the experiment. The experimental procedures were approved by the Animal Care and Ethics Committee of the University of New South Wales.

\section{Surgery and histology}

Approximately 10 min before surgery rats were injected intraperitoneally (i.p.) with $1.3 \mathrm{~mL} / \mathrm{kg}$ of the anaesthetic ketamine (CenVet Australia Pty Ltd.) at a concentration of $100 \mathrm{mg} / \mathrm{mL}$, and with the muscle relaxant xylazine $(0.3 \mathrm{~mL} / \mathrm{kg}$; Rompun; Bayer) at a concentration of $20 \mathrm{mg} / \mathrm{mL}$, and subcutaneously (s.c.) with $5 \mathrm{mg} / \mathrm{kg}$ carprofen. Each rat was then placed in a stereotaxic frame (Model 900; Kopf) with the incisor bar maintained at $\sim 3.3 \mathrm{~mm}$ below horizontal to achieve a flat skull position. A 30 -gauge needle attached to a 1- $\mu \mathrm{L}$ Hamilton microsyringe was lowered into the right AcbSh (A-P, 1.5; M-L, 0.85; D-V, -7.75; all distances in millimeters from bregma). Three hundred nanoliters of NMDA (Sigma-Aldrich) dissolved at $10 \mathrm{mg} / \mathrm{mL}$ in $0.1 \mathrm{M}$ phosphate buffered saline (PBS) pH 7.2 was injected over 5 min and the needle was left in place for $2 \mathrm{~min}$ prior to removal to allow for diffusion. Sham-operated rats underwent the same procedures but were injected with $0.1 \mathrm{M}$ PBS $\mathrm{pH} 7.2$ without NMDA. Immediately after surgery, rats were injected intramuscularly (i.m.) with $0.3 \mathrm{~mL}$ of a $300 \mathrm{mg} / \mathrm{mL}$ solution of procaine penicillin and $0.1 \mathrm{~mL}$ of a $100 \mathrm{mg} / \mathrm{mL}$ cephazolin sodium. Rats were given 5 $\mathrm{d}$ to recover from surgery before experimentation commenced.

NeuN immunohistochemistry was used to determine the location and the extent of the AcbSh lesion. At the conclusion of the experiments rats were deeply anesthetized with sodium pentobarbital (100 mg/kg i.p.) and perfused transcardially with $50 \mathrm{~mL}$ of $0.9 \%$ saline, containing $1 \%$ sodium nitrite and heparin (5000 i.u./mL), followed by $400 \mathrm{~mL}$ of $4 \%$ paraformaldehyde in $0.1 \mathrm{M}$ phosphate buffer (PB), $\mathrm{pH}$ 7.4. Brains were post-fixed for $1 \mathrm{~h}$ in the same fixative and placed in $20 \%$ sucrose solution overnight. Brains were blocked using a matrix aligned to the atlas of Paxinos and Watson (1997) and 40- $\mu \mathrm{m}$ coronal sections were cut using a cryostat (Microm HM560, Microm International) and stored in $0.1 \%$ sodium azide in $0.1 \mathrm{M}$ PBS pH 7.2. Sections were washed repeatedly in $0.1 \mathrm{M}$ PBS ( $\mathrm{pH} 7.2$ ), followed by a 2-h incubation in PBS ( $\mathrm{pH} 7.2$ ) containing 10\% natural horse serum (NHS) and $0.5 \%$ Triton X-100. Sections were then incubated in a mouse anti-serum against NeuN (1:500; Chemicon) and diluted in $0.1 \mathrm{M}$ PBS (pH 7.2; $0.1 \%$ sodium azide, $2 \%$ NHS, $0.2 \%$ Triton $\mathrm{X}-100$ (PBST-X with $0.1 \%$ sodium azide) for $48 \mathrm{~h}$ at room temperature with gentle agitation. After washing, sections were incubated for $4 \mathrm{~h}$ at room temperature in biotinylated donkey antimouse IgG (1:200; Jackson Immunoresearch Laboratories), diluted in $2 \%$ NHS PBT-X. After washing, sections were further incubated for $1 \mathrm{~h}$ in streptavidin CY3 (1:2000; Sigma). Sections were mounted and cover slipped with buffered glycerol ( $\mathrm{pH} 8.6)$.
Excitotoxic lesions of the AcbSh were determined by the absence of immunoreactivity for the neuronal marker NeuN. The size and location of the lesions, as defined by Paxinos and Watson (1997), were examined under a fluorescent microscope (Olympus BX-51) on sections spaced $160 \mu \mathrm{m}$ apart.

\section{Apparatus}

The apparatus consisted of a set of four identical chambers $(24 \mathrm{~cm}$ in length, $30 \mathrm{~cm}$ in width, and $21 \mathrm{~cm}$ in height). The front and rear walls, as well as the hinged lid, were constructed of clear Perspex and the end walls were made of stainless steel. The floor consisted of stainless steel rods, $4 \mathrm{~mm}$ in diameter, spaced $15 \mathrm{~mm}$ apart (center-to-center). Each chamber stood $2 \mathrm{~cm}$ above a tray of paper pellet bedding (Fibercycle). The chambers were cleaned with water, and the bedding underneath the chambers was changed between rats. These chambers were located individually within sound-attenuating boxes that were painted white. The boxes were illuminated by infrared light emitting diodes. Illumination within the conditioning chambers was $15 \mathrm{~cd} / \mathrm{m}^{2}$. Digital video cameras were mounted on the rear wall of each box and connected to a digital multiplexer in an adjacent room that, in turn, was connected to a digital video disc recorder. The stimuli used for conditioning were controlled by computer (LabView, National Instruments).

In Experiment 1, CSs A, B, and C were two auditory and two visual CSs. The two auditory CSs consisted of an 82-dB (A scale) $750-\mathrm{Hz}$ tone and an $82-\mathrm{dB}$ (A scale) $20-\mathrm{Hz}$ clicker generated digitally and delivered through speakers mounted in the ceiling of each box. The two visual CSs consisted of a constant or flashing (4-Hz) presentation of a white fluorescent light producing an illumination level of $75 \mathrm{~cd} / \mathrm{m}^{2}$ within the chambers. The light was mounted on the ceiling of each box, immediately above the conditioning chamber. For half the animals in each group, the visual stimuli (light or flash) served as CSA with the auditory stimuli (clicker and tone) counterbalanced across animals as CSB and CSC. For the other half of the animals, the roles of the auditory and visual stimuli were interchanged. All CSs were $30 \mathrm{sec}$ in duration, and during conditioning they coterminated with the footshock US. This US was a $1 \mathrm{sec}, 0.4 \mathrm{~mA}$ unscrambled AC $50 \mathrm{~Hz}$ shock from a constant-current generator that was delivered to the floor of each chamber.

In Experiment 2 there were two visual CSs (light or flash) and two auditory CSs (clicker or tone) previously described. For half of the animals in each group, the visual stimuli (light and flash) served as CSA and CSB with the auditory stimuli (clicker and tone) counterbalanced across animals as CSC and CSD. For the other half of the animals, the roles of the auditory and visual stimuli were interchanged. The US was an unscrambled AC $50-\mathrm{Hz}$ shock 1 sec in duration and a $0.4 \mathrm{~mA}$ intensity from a constantcurrent generator that was delivered to the floor of each chamber.

\section{Behavioral Procedures}

\section{Experiment 1}

\section{Pre-exposure}

On Days 1 and 2, all rats were placed in conditioning chambers for 11 min. During this session, rats received four nonreinforced 30-sec presentations of each CS at an interstimulus interval (ISI) of $30 \mathrm{sec}$. For each experiment, all pre-exposure, conditioning, and test sessions began with a 3-min preCS period during which no stimuli were presented. Rats were pre-exposed to the CSs to encourage discrimination between them (Mackintosh and Bennett 1998).

\section{Stage I}

Stage I training occurred on Days 3 and 4 and rats were placed in the conditioning chambers for approximately 20 min. During 
each session, they received four pairings of CSA with the US. The ITI was random about a mean of $240 \mathrm{sec}$.

\section{Stage II}

Stage II training occurred on Days 5, 6, and 7. Rats were placed in conditioning chambers for approximately $45 \mathrm{~min}$. During this session they received four pairings of compound $\mathrm{AB}$ with the 1-sec 0.4-mA footshock and four nonreinforced presentations of compound $\mathrm{AC}$. $\mathrm{AB}$ and $\mathrm{AC}$ were presented in a counterbalanced order within subjects. For all CS presentations the ITI was random about a mean of $240 \mathrm{sec}$. Five hours after each reinforced session, rats were placed in the conditioning chambers for $10 \mathrm{~min}$ and no stimuli were delivered. These exposures were intended to reduce the levels of background or context fear.

\section{Test}

On Day 8, all rats were tested for their fear reactions to compound $\mathrm{CSs} \mathrm{AB}$ and $\mathrm{AC}$. These sessions were $16 \mathrm{~min}$ in duration, and rats received three nonreinforced presentations of each 30 -sec compound $\mathrm{CS}$ in the order $\mathrm{AB}, \mathrm{AC}, \mathrm{AC}, \mathrm{AB}, \mathrm{AC}$, and $\mathrm{AB}$. The ISI was $30 \mathrm{sec}$.

\section{Experiment 2}

\section{Pre-exposure}

The procedure for pre-exposure was identical to that described for Experiment 1.

\section{Stage I}

Stage I training occurred on Days 3 and 4 . Rats were placed in the conditioning chambers for approximately $20 \mathrm{~min}$. During this session they received two pairings of CSA and CSC with the US. The order of CSA and CSC presentations was counterbalanced within subjects. The ITI was random about a mean of $240 \mathrm{sec}$.

\section{Stage II}

Stage II training occurred on Days 5 and 6. Rats were placed in the conditioning chambers for $8 \mathrm{~min}$. They received one presentation of the 30-sec $\mathrm{AB}$ compound on each day followed by the US. Five hours after each reinforced session rats were placed in the chambers for $10 \mathrm{~min}$ to reduce fear of the context.

\section{Test}

On Day 7 all rats were tested for their fear reactions to nonreinforced presentations of the compounds $\mathrm{AD}$ and $\mathrm{BC}$. These sessions were $11 \mathrm{~min}$ in duration and consisted of four presentations of each 30-sec compound in the following order: $\mathrm{AD}, \mathrm{BC}, \mathrm{BC}, \mathrm{AD}$, $\mathrm{BC}, \mathrm{AD}, \mathrm{AD}$, and $\mathrm{BC}$. The ISI was $30 \mathrm{sec}$.

\section{Scoring and statistics}

Performance during all stages was recorded. Freezing, defined as the absence of all movement other than that required for respiration (Fanselow 1980), was scored every 2 sec during the 3-min preCS period and during the $30 \mathrm{sec}$ of each CS presentation. The records of each rat were scored by two observers, one of whom was unaware of each rat's group allocation. The inter-rater reliability or the correlation between the percentages of observations scored as freezing for each rat between observers, exceeded 0.93 in all experiments. The percentages of observations of freezing were then analyzed by means of a planned orthogonal contrast testing procedure. The decision-wise error rate $(\alpha)$ was controlled at the 0.05 level for each contrast tested using the procedure described by Hays (1972).

\section{Acknowledgments}

This research was supported by an Australian Postgraduate Award to L.A.B. and by a grant from the Australian Research Council to G.P.M. (DP0877430). G.P.M. is an Australian Research Council QEII Research Fellow (DP0877430).

\section{References}

Bradfield L, McNally GP. 2008. Unblocking in Pavlovian fear conditioning. J Exp Psychol Anim B 34: 256-265.

Cassaday HJ, Horsley RR, Norman C. 2005. Electrolytic lesions to nucleus accumbens core and shell have dissociable effects on conditioning to discrete and contextual cues in aversive and appetitive procedures respectively. Behav Brain Res 160: 222-235.

Day JJ, Carelli RM. 2007. The nucleus accumbens and reward learning. Neuroscientist 13: 148-159.

Delgado MR, Li J, Schiller D, Phelps EA. 2008. The role of the striatum in aversive learning and aversive prediction errors. Philos Trans $R$ Soc Lond B Biol Sci 363: 3787-3800.

Deutch AY, Cameron DS. 1992. Pharmacological characterization of dopamine systems in the nucleus accumbens core and shell. Neuroscience 46: 325-328.

Fanselow MS. 1980. Conditional and unconditional components of post-shock freezing. Pavlovian J Biol Sci 15: 177-182.

Fanselow MS, Poulos AM. 2005. The neuroscience of mammalian associative learning. Annual Rev Psychol 56: 207-234.

Gal G, Schiller D, Weiner I. 2005. Latent inhibition is disrupted by nucleus accumbens shell lesion but is abnormally persistent following entire nucleus accumbens lesion: The neural site controlling the expression and disruption of the stimulus preexposure effect. Behav Brain Res 162: 246-255.

Groenewegen HJ, Vermeulen-Van Der Zee E, Te Kortschot A, Witter MP. 1987. Organization of the projections from the subiculum to the ventral striatum in the rat: A study using anterograde transport of Phaseolus vulgaris leucoagglutinin. Neuroscsience 23: 103-120.

Han J-S, Gallagher M, Holland PC. 1995. Hippocampal lesions disrupt decrements but not increments in conditioned stimulus processing. $J$ Neurosci 15: 7323-7329.

Hays WL. 1972. Statistics for the social sciences. Holt, Rinehart \& Winston, New York.

Jongen-Relo AL, Kaufmann S, Feldon J. 2003. A differential involvement of the shell and core subterritories of the nucleus accumbens of rats in memory processes. Behav Neurosci 117: 150-168.

Josselyn SA, Falls WA, Gewirtz JC, Pistell P, Davis M. 2005. The nucleus accumbens is not critically involved in mediating the effects of a safety signal on behavior. Neuropsychopharmacology 30: 17-26.

Kalivas PW, Duffy P. 1995. Selective activation of dopamine transmission in the shell of the nucleus accumbens by stress. Brain Res 675: 325-328.

Kaye H, Pearce JM. 1987. Hippocampal lesions attenuate latent inhibition and the decline of the orienting response in rats. QJ Exp Psychol 39B: $107-125$.

Knutson B, Cooper JC. 2005. Functional magnetic imaging of reward prediction. Current Opin Neurol 18: 411-417.

Mackintosh NJ, Bennett CH. 1998. Perceptual learning in animals and humans. In Advances in psychological science: Vol 2. Biological and cognitive aspects (ed. M Sabourin, F Craik, M Roberts), pp. 317-333. Psychology Press, Hove, UK.

Maldonado-Irizarry CS, Kelley AE. 1995. Excitotoxic lesions of the core and shell subregions of the nucleus accumbens differentially disrupt body weight regulation and motor activity in the rat. Brain Res Bull 38: $551-559$.

Maren S. 2005. Building and burying fear memories in the brain. Neuroscientist 11: 89-99.

McNally GP, Westbrook RF. 2006. A short intertrial interval facilitates acquisition of context-conditioned fear and a short retention interval facilitates its expression. J Exp Psychol: Anim B 32: 164-172.

Mogenson GJ. 1987. Limbic-motor integration. In Progress in psychobiology and physiological psychology, Vol. 12, pp. 117-170. Academic Press, New York.

Pare D, Quirk GJ, LeDoux JE. 2004. New vistas on amygdala networks in conditioned fear. J Neurophysiol 92: 1-9.

Parkinson JA, Robbins TW, Everitt BJ. 1999. Selective excitotoxic lesions of the nucleus accumbens core and shell differentially affect aversion Pavlovian conditioning to discrete and contextual cues. Psychobiology 27: $256-266$. 
Paxinos G, Watson C. 1997. The rat brain in stereotaxic coordinates. Elsevier Academic, Amsterdam, The Netherlands.

Rescorla RA. 2001. Unequal associative changes when excitors and neutral stimuli are conditioned in compound. Q J Exp Psychol B 54: 53-68.

Riedel G, Harrington NR, Hall G, MacPhail EM. 1997. Nucleus accumbens lesions impair context, but not cue, conditioning in rats. Neuroreport 8: $2477-2481$.

Seymour B, O'Doherty JP, Dayan P, Koltzenburg M, Jones AK, Dolan RJ, Friston KJ, Frackowiak RS. 2004. Temporal difference models describe higher-order learning in humans. Nature 429: 664-667.

Weiner I, Gal G, Rawlins JNP, Feldon J. 1996. Differential involvement of the shell and core subterritories of the nucleus accumbens in latent inhibition and amphetamine-induced activity. Behav Brain Res 81: $123-133$.

Weiner I, Gal G, Feldon J. 1999. Disrupted and undisruptable latent inhibition following shell and core lesions. Annals NY Acad Sci 877: $723-727$

Westbrook RF, Good AJ, Kiernan MJ. 1997. Microinjection of morphine into the nucleus accumbens impairs contextual learning in rats. Behav Neurosci 111: 996-1013.

Wise RA. 2006. Role of brain dopamine in food reward and reinforcement. Philos Trans R Soc Lond B Biol Sci 361: 1149-1158.

Received March 8, 2010; accepted in revised form May 17, 2010. 


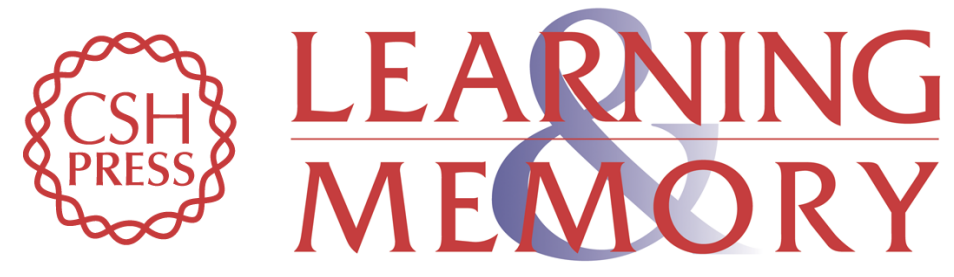

\section{The role of nucleus accumbens shell in learning about neutral versus excitatory stimuli during Pavlovian fear conditioning}

Laura A. Bradfield and Gavan P. McNally

Learn. Mem. 2010, 17:

Access the most recent version at doi:10.1101//m.1798810

References This article cites 28 articles, 1 of which can be accessed free at:

http://learnmem.cshlp.org/content/17/7/337.full.html\#ref-list-1

License

Email Alerting Receive free email alerts when new articles cite this article - sign up in the box at the Service top right corner of the article or click here. 